\title{
Surface atomic properties of tetrahedral amorphous carbon
}

\author{
C.W. Chen, J. Robertson * \\ Department Materials Science and Engineering, National Taiwan University, Taipei, Taiwan \\ Department of Engineering, University of Cambridge, Cambridge CB2 1PZ, UK
}

Available online 6 January 2006

\begin{abstract}
The surface energy and surface atomic structure of tetrahedral amorphous carbon has been calculated by an ab-initio method. The surface atoms are found to reconstruct into $\mathrm{sp}^{2}$ sites often bonded in graphitic rings. Placing the dangling bonds on adjacent surface atoms lower their energy by $\pi$-bonding and this is the source of the low surface energy. The even lower surface energy of hydrogenated amorphous carbon (a-C:H) is due to the hydrogenation of all broken surface bonds.

(C) 2005 Elsevier B.V. All rights reserved.
\end{abstract}

Keywords: Diamond-like carbon; Surface; Electronic structure; Calculations

\section{Introduction}

Diamond-like carbon (DLC) is amorphous carbon with a high $\mathrm{sp}^{3}$ content. It generally has a low surface energy and DLC has previously been described as a ceramic with low surface energy [1]. Recently, alloys of DLC with metals have been created to increase its toughness, while it has been alloyed with non-metals such as $\mathrm{F}, \mathrm{O}, \mathrm{N}$ and $\mathrm{Si}$ to modify its surface energy $[1,2]$.

There are two forms of DLC, hydrogenated amorphous carbon $(\mathrm{a}-\mathrm{C}: \mathrm{H})$ usually prepared from a hydrocarbon precursor and hydrogen-free amorphous carbon $(\mathrm{a}-\mathrm{C})$. One variety of a-C with a very high $\mathrm{sp}^{3}$ content is called tetrahedral amorphous carbon (ta-C).

DLC is widely used as a protective coating for read heads and disks in magnetic storage technology [3], and is being developed for use as an alternative material for use in micro electromechanical systems (MEMS) [3-7]. One of the interesting properties of DLC for MEMS compared to the more usual $\mathrm{Si}$ is that it is hydrophobic, so that its surfaces do not suffer from stiction and its MEMS surfaces are easier to separate. DLC is also of interest as a biocompatible coating [7]. Here again, the surface properties and controllable hydrophobicity is of importance in controlling cell attachment. The low

\footnotetext{
* Corresponding author. Tel.: +44 1223 332689; fax: +44 1223332662.

E-mail address: jr@eng.cam.ac.uk (J. Robertson).
}

surface energy of a-C:H is also of interest in its friction properties [8].

The cause of the low surface energy of a-C:H can be attributed to the $\mathrm{C}-\mathrm{H}$ bonding on its surface. On the other hand, the low surface energy of ta- $\mathrm{C}$ is less obvious, as it only has $\mathrm{C}-\mathrm{C}$ bonds. This is the subject of this paper. Here we take a relaxed network of ta- $\mathrm{C}$ and cleave it into two halves, relax the surface and calculate the surface energy. There have been a number of previous studies of the atomic structure of ta-C surfaces [9-12], but so far no calculation of its surface energy.

\section{Methods}

A random network of 200 atoms of ta-C is created as a periodic supercell by quenching a random array of atoms. The lattice constant of the cell is set to give a density of $3.0 \mathrm{~g} / \mathrm{cm}^{3}$. The structure is relaxed by ab-initio methods using the CASTEP total energy plane wave pseudopotential code [13]. The carbon is represented by ultra-soft pseudopotentials, with a plane wave cutoff of $200 \mathrm{eV}$ and the PBE electronic exchangecorrelation functional.

The resulting bulk ta-C network has a fraction of 4-fold coordinated $\mathrm{sp}^{3}$ sites of $73 \%$, using as bond length cut off of $1.79 \AA$. The overall numbers are $1 \% \mathrm{sp}^{1}: 26 \% \mathrm{sp}^{2}: 73 \% \mathrm{sp}^{3}$. This cell has a lattice constant in Oz of $17.5 \AA$.

The lattice is cleaved into two halves of 100 atoms in the $\mathrm{Oz}$ direction and a vacuum gap is introduced. The cell length increased to $30 \AA$. The resulting surfaces are allowed to relax to 


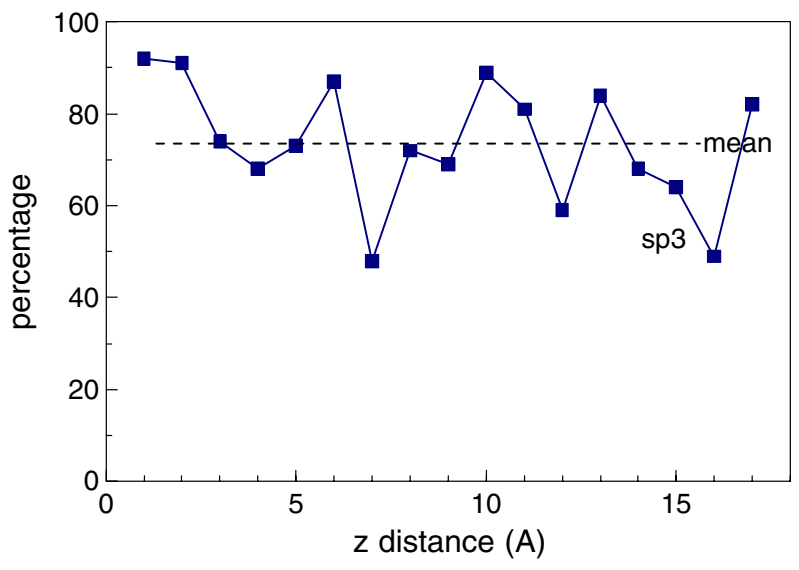

Fig. 1. $\mathrm{sp}^{3}$ profile along $\mathrm{Oz}$ direction of the bulk supercell of ta-C.

minimise their total energy. The atomic coordinations are calculated. The increase in total energy is assigned as a surface energy.

\section{Results and discussion}

The coordination of the networks is analysed by plotting the fraction of atoms of coordinations 2,3 and 4 in $1 \AA$ slices perpendicular to the surface. Fig. 1 plots the $\mathrm{sp}^{3}$ fraction of the original uncleaved network, showing the average $\mathrm{sp}^{3}$ fraction of $73 \%$.

Fig. 2 shows the $\mathrm{sp}^{3}$ fraction for one of the cleaved units. The $\mathrm{sp}^{3}$ fraction at the centre of the network averages $75 \%$, similar to that in the initial large network, leaving about $25 \%$ $\mathrm{sp}^{2}$ sites. It is seen that the $\mathrm{sp}^{2}$ content rises at the surface to nearly $90 \%$. Thus, the surface atoms are almost entirely $\mathrm{sp}^{2}$ coordinated. The transition to $\mathrm{sp}^{2}$ bonding occurs within $1 \mathrm{~A}$ or one atomic layer thickness. Thus, the network is essentially $\mathrm{sp}^{3}$ coordinated, except for the $\mathrm{sp}^{2}$ coordinated surface. Prior to relaxation, the surface atoms had dangling bonds. These atoms reconstructed, as at the surface of a crystal, to become $\mathrm{sp}^{2}$. The increase in total energy from forming the surface gives a surface energy of $0.116 \mathrm{eV} / \mathrm{A}^{2}$. This corresponds to $1.9 \mathrm{~J} / \mathrm{m}^{2}$.

On a diamond (111) surface, each surface atom has three bonds and one dangling bond. On the ideal surface, the

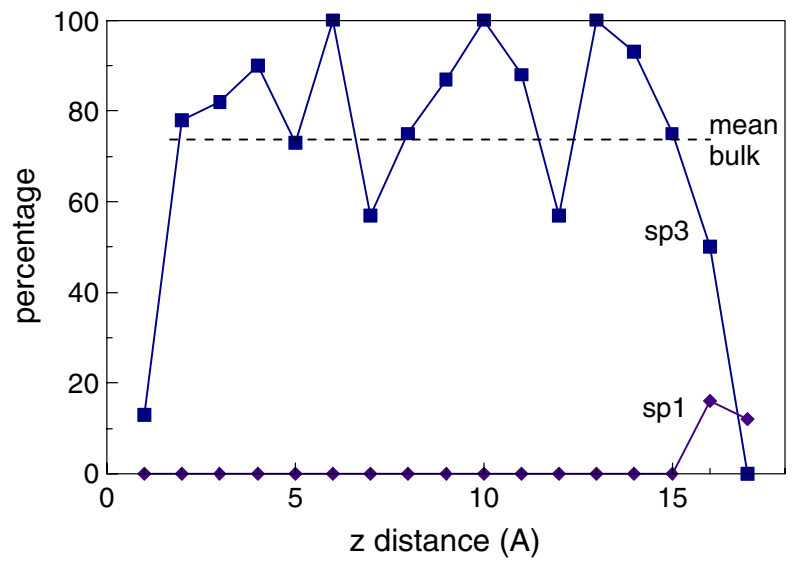

Fig. 2. $\mathrm{sp}^{3}$ profile along $\mathrm{Oz}$ direction of the ta-C supercell with surfaces.

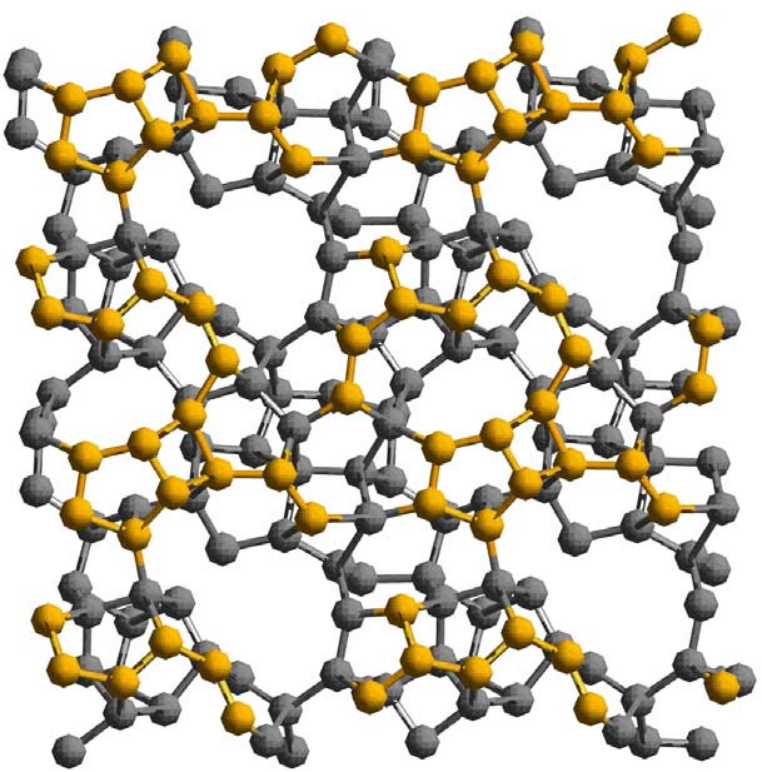

Fig. 3. Calculated atomic structure of a ta-C surface, with the surface atoms in yellow. (For interpretation of the references to colour in this figure legend, the reader is referred to the web version of this article.)

dangling bonds are on second neighbour atoms. This surface reconstructs so that the dangling bonds become nearest neighbour atoms, in the so-called $\pi$-bonded chain [14], a bit like in poly-acetylene. An ideal diamond (100) surface consists of atoms with two dangling bonds [14]. This surface reconstructs so as to pair up adjacent atoms, so that they form a lateral bond between them. This leaves one dangling bond per atom on adjacent atoms. The dangling bonds actually form a set of isolated $\pi$-bonds. Thus, both major surfaces of diamond consist of reconstructions with $\pi$-bonded dangling bonds on adjacent atoms.

Fig. 3 shows the surface atomic structure of one of the two surfaces formed in our calculation. Forming a surface is expected to cut bonds, so it is no surprise that the surface atoms

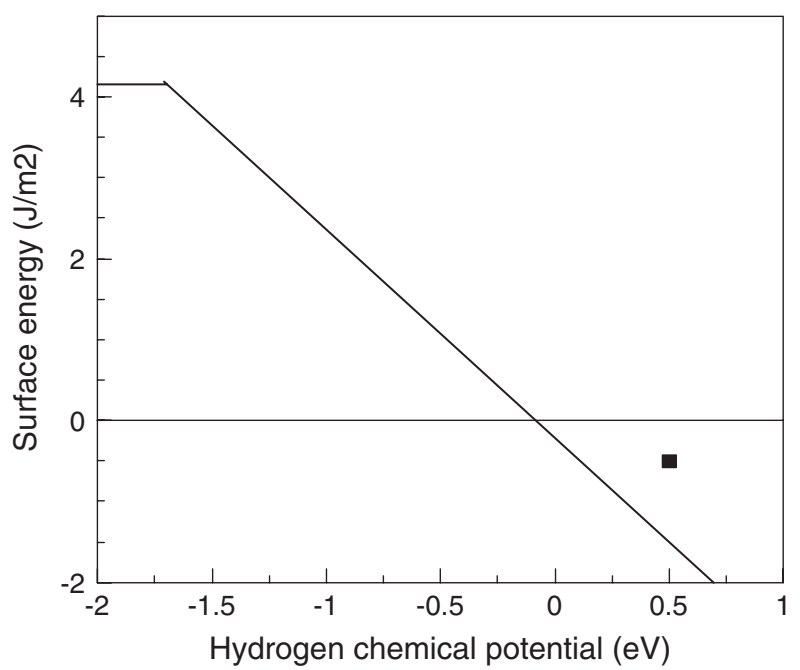

Fig. 4. Calculated surface energy of the hydrogenated diamond (111) surface vs. hydrogen chemical potential, showing negative surface energy at high chemical potential. Zero chemical potential corresponds to the activity of $\mathrm{H}$ in a methane molecule. After Hong and Chou [20]. 
shown in yellow are largely $\mathrm{sp}^{2}$ bonded. Nevertheless, the surface atoms have rearranged themselves to be mainly nearest neighbours and many of them have formed into six-fold rings of $\mathrm{sp}^{2}$ atoms. Thus, the surface is trying to form graphitic rings. A similar structure was also previously found in simulations by Dong and Drabold [9], Kelires [10] and Haerle et al. [11].

This reconstruction is the basis of the low surface energy of ta-C. The surface atoms are forming $\pi$-bonded orbitals on adjacent atoms, as in the diamond surface. However, on ta-C, they form the more stable graphitic rings, rather than $\pi$-bonded chains. Thus, the surface energy of ta-C is lower than of bare diamond (100) or (111) surfaces.

The existence of an $\mathrm{sp}^{2}$ bonded, reconstructed, outer atomic layer should not be confused with a more general low-density layer on real ta-C surfaces. ta-C is usually formed by ion beam deposition, at ion energies of 25 to $100 \mathrm{eV}$. The ion energy is thought to be the origin of the $\mathrm{sp}^{3}$ bonding via the mechanism of subplantation [15]. It is known from EELS measurements [16] that the surface of a ta-C film will consist of a 0.2 to $1 \mathrm{~nm}$ thick layer of lower density and enhanced $\mathrm{sp}^{2}$ bonding. The thickness equals the ion range and depends on the ion energy. This occurs because the subplantation mechanism does not densify the layers within the ion range. This $\mathrm{sp}^{2}$-rich layer is different from the layer in the present calculations. The layer in the calculations is 1 atom thick and will exist because of the cutting of bonds. The layer in the real ta-C films exists because of the subplantation mechanism. It can be etched off by an oxygen plasma [17], leaving the bulk $\mathrm{sp}^{3}$. However, the last atomic layer here cannot be converted to $\mathrm{sp}^{3}$ by etching. The surface electronic structure of ta-C has been measured by photoemission by Reinke et al. [18] and shows the strong $\mathrm{sp}^{2}$ bonding.

Our calculated surface energy of ta-C compares to the surface fraction energy $[19,20]$ of diamond of $5.3 \mathrm{~J} / \mathrm{m}^{2}$. Experimentally, the surface energy of ta-C is about $0.1 \mathrm{~J} / \mathrm{m}^{2}$ and that of a-C:H is $0.08 \mathrm{~J} / \mathrm{m}^{2}$, which can be reduced to as low as $0.04 \mathrm{~J} / \mathrm{m}^{2}$ by alloying with $\mathrm{F}$ or $\mathrm{Si}[1]$.

The cause of the low surface energy of a-C:H or of the hydrogenated surface of diamond is different. The bond energies of $\mathrm{C}-\mathrm{C}, \mathrm{H}-\mathrm{H}$ and $\mathrm{C}-\mathrm{H}$ bonds are $4.29,3.7$ and $4.51 \mathrm{eV}$, respectively. Cleaving a (111) diamond surface leaving it terminated by $\mathrm{C}-\mathrm{H}$ bonds, in effect occurs by the reaction

$\mathrm{C}-\mathrm{C}+\mathrm{H}-\mathrm{H} \rightarrow 2 \mathrm{C}-\mathrm{H}$

This reaction is exothermic by $1.0 \mathrm{eV}$. Thus, the surface energy calculated this way would be negative. Indeed, the formation energy of a hydrogenated (111) diamond surface has been calculated as a function of hydrogen chemical potential and is shown in Fig. 4. It shows a negative surface energy for high hydrogen chemical potential $\mu_{\mathrm{H}}$.

A similar effect will occur on the a-C:H surface. It is assumed that all potential dangling bonds have become saturated as $\mathrm{C}-\mathrm{H}$ bonds. Thus, the low surface energy of a$\mathrm{C}: \mathrm{H}$ and of other monovalent terminations like a-C:F is entirely chemical. On the other hand, the modifications to surface energy caused by network modifying additions such as $\mathrm{O}$ or $\mathrm{Si}$ is more complex and may result from a change of $\mathrm{sp}^{3}$ content or hydration properties of the surface in the case of $\mathrm{Si}$.

\section{References}

[1] M. Grischke, A. Hieke, F. Morgenweck, H. Dimigen, Diamond Relat. Mater. 7 (1998) 454.

[2] B.K. Tay, Y.H. Cheng, X.Z. Ding, Diamond Relat. Mater. 10 (2000) 1082; A.A. Voevodin, M.A. Capano, S.J.P. Laube, M.S. Donley, J.S. Zabinski, Thin Solid Films 298 (1997) 107;

A.A. Voevodin, S.V. Prasad, J.S. Zabinski, J. Appl. Phys. 82 (1997) 855; S. Zhang, et al., Thin Solid Films 482 (2005) 138.

[3] J.P. Sullivan, T.A. Friedmann, K. Hjort, MRS Bull. 26 (April 2001) 309.

[4] S. Cho, I. Chasiotis, T.A. Friedmann, J. Micromechanics Microengineering 15 (2005) 728.

[5] H.W. Liu, B. Bhushan, J. Vac. Sci. Technol., A 21 (2003) 1528.

[6] D.H.C. Chua, W.I. Milne, D. Sheeja, B.K. Tay, D. Schneider, J. Vac. Sci. Technol., B 22 (2004) 2680.

[7] P.D. Maguire, J.A. McLaughlin, T.I.T. Okpalugo, P. Lemoine, P. Papakonstantinou, E.T. McAdams, M. Needham, A.A. Ogwu, M. Ball, G.A. Abbas, Diamond Relat. Mater. 14 (2005) 1277.

[8] A. Erdemir, Trib. Int. 37 (2004) 1005; Surf. Coat. Technol. 146 (2001) 292.

[9] D. Dong, D.A. Drabold, Phys. Rev., B 57 (1998) 15591.

[10] P.C. Kelires, J. Non-Cryst. Solids 230 (1998) 597.

[11] R. Haerle, G. Galli, A. Baldereschi, App. Phys. Lett. 75 (1999) 1718; J. Non Cryst. Solids 266 (2000) 740.

[12] M. Kaukonen, et al., Phys. Rev. Lett. 83 (1999) 5346.

[13] V. Milman, B. Winkler, J.A. White, C.J. Pickard, M.C. Payne, Int. J. Quantum Chem. 77 (2000) 895.

[14] J. Furthmuller, J. Hafner, G. Kresse, Phys. Rev., B 53 (1996) 7334; G. Kern, J. Hafner, G. Kresse, Surf. Sci. 352 (1996) 745; Surf. Sci. 455 (1996) 366

[15] J. Robertson, Diamond Relat. Mater. 2 (1993) 984; Diamond Relat. Mater. 3 (1994) 361; Mat. Sci. Rep. R. 37 (2002) 129.

[16] C.A. Davis, K.M. Knowles, G.A.J. Amaratunga, Phys. Rev. Lett. 80 (1998) 3280.

[17] F.J. Clough, W.I. Milne, B.Y. Kleinsorge, J. Robertson, G. Amaratunga, B.N. Roy, Electron. Lett. 32 (1996) 498.

[18] P. Reinke, et al., J. Electron Spectosc. Relat. Phenom. 136 (2004) 239.

[19] R.H. Telling, C.J. Pickard, M.C. Payne, J.E. Field, Phys. Rev. Lett. 84 (2000) 5160.

[20] S. Hong, M.Y. Chou, Phys. Rev., B 57 (1998) 6262. 